\title{
SOME PROPERTIES OF OPERATOR EXPONENT
}

\author{
Lyudmila F. Korkina ${ }^{\dagger}$ and Mark A. Rekant ${ }^{\dagger \dagger}$ \\ Ural Federal University, \\ 51 Lenin aven., Ekaterinburg, Russia, 620000 \\ †l.f.korkina@urfu.ru, ${ }^{\dagger} m . a . r e k a n t @ u r f u . r u$
}

\begin{abstract}
We study operators given by series, in particular, operators of the form $e^{B}=\sum_{n=0}^{\infty} B^{n} / n$ !, where $B$ is an operator acting in a Banach space $X$. A corresponding example is provided. In our future research, we will use these operators for introducing and studying functions of operators constructed (with the use of the Cauchy integral formula) on the basis of scalar functions and admitting a faster than power growth at infinity.
\end{abstract}

Keywords: Closed operator, Operator exponent, Multiplicative property.

The theory of functions of normal operators has been developed in Hilbert spaces [8, Ch. 12,13]. However, functions of an operator in Banach spaces are introduced under quite serious restrictions on the operator and the corresponding scalar functions (see e.g. [2, Ch. VII.3]). For a considerable class of operators, these scalar functions are assumed to be analytical with polynomial growth at infinity (see e.g. [1] and [6, Ch. $1, \S 5]$ ). The authors' papers [3-5] are in the same vein. In these papers, based on the Cauchy integral formula, functions of an operator were constructed in terms of natural powers of the operator. To introduce and study functions of an operator built constructed on the basis of scalar functions and admitting the growth at infinity faster than the power function but not faster than the exponential function have, we will need operators of the form

$$
e^{B}=\sum_{n=0}^{\infty} \frac{B^{n}}{n !},
$$

where $B$ is an operator on a Banach space $X$. In this paper, we study the properties of such operators.

We will use series of elements of a Banach space $X$ and operator series. The principal notions of numerical series (double series and repeated series) are naturally extended to series of elements of the space $X[7, \mathrm{Ch} .2, \S 2]$. In this paper, the convergence of partial sums of series from $\mathrm{X}$ is interpreted as the convergence in the norm of this space. For a series $\sum_{n=0}^{\infty} A_{n}$ of operators $A_{n}$ acting in $X$, its sum is the operator $A$ with the domain $\mathcal{D}(A)=\left\{x \in X: \sum_{n=0}^{\infty} A_{n} x\right.$ converges $\}$ and such that $A x=\sum_{n=0}^{\infty} A_{n} x$ for $x \in \mathcal{D}(A)$. The expression $A \subset B(B \supset A)$ for operators $A$ and $B$ means that $B$ is the extension of $A[7$, Ch. 7 , Sect. 6].

Let us proceed to the results.

In what follows, we will need the following auxiliary assertion.

Assertion 1. The following statements hold:

(i) (An analog of Abel's test for numerical series). Suppose that a series $\sum_{n=0}^{\infty} a_{n}$ converges in $X$ and a sequence $\left\{\alpha_{n}\right\}_{n=0}^{\infty} \subset \mathbb{R}$ is monotonic and bounded. Then, the series $\sum_{n=0}^{\infty} \alpha_{n} a_{n}$ converges. 
(ii) Suppose that $\left\{a_{m, n}\right\}_{m, n=0}^{\infty} \subset X$ and the series $\sum_{m, n=0}^{\infty} a_{m, n}$ converges absolutely. Then, every rearrangement of this series converges absolutely to the same sum.

(iii) Suppose that the terms of a series $\sum_{m, n=0}^{\infty} a_{m, n}\left(a_{m, n} \in X\right)$ are reindexed (with a single index) and the series $\sum_{k=0}^{\infty} b_{k}$ is composed of them. If one of these two series or the repeated series $\sum_{m=0}^{\infty} \sum_{n=0}^{\infty} a_{m, n}$ converges absolutely, then the other two series converge absolutely to the same sum.

(iv) If a series $\sum_{m, n=0}^{\infty} a_{m, n}\left(a_{m, n} \in X\right)$ converges absolutely, then the series $\sum_{k=0}^{\infty} \sum_{\substack{m+n=k \\(m, n \geq 0)}}^{\infty} a_{m, n}$ also converges absolutely to the same sum.

The proof of statement $(i)$ is almost the same as the proof of Abel's test for numerical series. The proofs of statements $(i i)-(i v)$ reduce to the use of the corresponding statements for numerical series after the application a continuous linear functional to the series under consideration. Here, we take into account the fact that if values of all such functionals coincide at two elements from $X$, then these elements are equal [2, Ch. II.3.15].

Assertion 2. Suppose that $A$ is an operator acting in $X, x \in X, k \in \mathbb{N}$, a sequence $\left\{\alpha_{n}\right\}_{n=0}^{\infty} \subset \mathbb{R}$ is such that the sequence $\left\{\frac{\alpha_{n+k}}{\alpha_{n}}\right\}$ is monotonic and bounded, and the series $\sum_{n=0}^{\infty} \alpha_{n} A^{n+k} x$ converges. Then, the series $\sum_{n=0}^{\infty} \alpha_{n} A^{n} x$ converges. If the operator $A^{k}$ is linear and closed, then the following equality holds:

$$
\sum_{n=0}^{\infty} \alpha_{n} A^{n+k} x=A^{k} \sum_{n=0}^{\infty} \alpha_{n} A^{n} x
$$

which is equivalent to the expression

$$
\sum_{n=0}^{\infty} \alpha_{n} A^{n+k} \subset A^{k} \sum_{n=0}^{\infty} \alpha_{n} A^{n}
$$

P r o o f. The following relations are valid:

$$
\sum_{n=0}^{\infty} \alpha_{n} A^{n} x=\sum_{n=0}^{k-1} \alpha_{n} A^{n} x+\sum_{n=0}^{\infty} \alpha_{n+k} A^{n+k} x=\sum_{n=0}^{k-1} \alpha_{n} A^{n} x+\sum_{n=0}^{\infty} \frac{\alpha_{n+k}}{\alpha_{n}}\left(\alpha_{n} A^{n+k} x\right)
$$

The latter series converges by the analog of Abel's test. Moreover, under the assumption that the operator $A^{k}$ is linear and closed, equality (2) holds.

Remark 1. The operator $A^{k}$ is linear and closed if the operator $A$ is linear and its resolvent set $\rho(A) \neq \varnothing[2$, VII.9.7].

Remark 2. The boundedness and monotonicity of the sequence $\left\{\frac{\alpha_{n+k}}{\alpha_{n}}\right\}$ starting from a certain index follow from the fact that the sequence $\left\{\frac{\alpha_{n+1}}{\alpha_{n}}\right\}$ is monotonic and bounded.

This remark follows from the relation

$$
\frac{\alpha_{n+k}}{\alpha_{n}}=\frac{\alpha_{n+1}}{\alpha_{n}} \times \frac{\alpha_{n+2}}{\alpha_{n+1}} \times \cdots \times \frac{\alpha_{n+k}}{\alpha_{n+k-1}}
$$


and the fact that terms of a monotonic sequence of reals are of the same sign starting from a certain index.

Remark 3. Equality (2) holds without the assumption that the sequence $\left\{\frac{\alpha_{n+k}}{\alpha_{n}}\right\}$ is monotonic and bounded if the operator $A^{k}$ is linear and closed and both the series in (2) converge.

Corollary 1. Suppose that $k \in \mathbb{N}$ and the operator $A^{k}$ is linear and closed. Then,

$$
e^{A} A^{k} \subset A^{k} e^{A} \text {. }
$$

To prove this fact, it is sufficient to take $\alpha_{n}=1 / n$ ! in (3).

In Assertion 1, the sequence $\left\{\frac{\alpha_{n+k}}{\alpha_{n}}\right\}_{n=0}^{\infty}$ is required to be monotonic and bounded. Let us consider the conditions related to these properties.

Lemma 1. If $a \in \mathbb{R}$ and a sequence $\left\{\alpha_{n}\right\} \subset(0,+\infty)$ is such that $\frac{\alpha_{n+1}}{\alpha_{n}} \leq$ a for all $n$, then $\alpha_{n} \leq C a^{n}$ for all $n$, where $C=\alpha_{1} / a$. Conversely, if the sequence $\left\{\frac{\alpha_{n+1}}{\alpha_{n}}\right\}$ is monotonic and $\alpha_{n} \leq C a^{n}$ for some $C, a \in(0,+\infty)$ and all $n$, then the sequence $\left\{\frac{\alpha_{n+1}}{\alpha_{n}}\right\}$ is bounded.

P r o o f. Suppose that $a \in \mathbb{R}$ is such that $\frac{\alpha_{n+1}}{\alpha_{n}} \leq a$ for all $n$. Then,

$$
\frac{\alpha_{n}}{\alpha_{1}}=\frac{\alpha_{2}}{\alpha_{1}} \times \frac{\alpha_{3}}{\alpha_{2}} \times \cdots \times \frac{\alpha_{n}}{\alpha_{n-1}} \leq a^{n-1}
$$

i. e., $\alpha_{n} \leq C a^{n}$ for $C=\alpha_{1} / a$.

Conversely, suppose that $\alpha_{n} \leq C a^{n}$ for some $C, a \in(0,+\infty)$, and all $n$ and the sequence $\left\{\frac{\alpha_{n+1}}{\alpha_{n}}\right\}$ is monotonic. Denote by $d$ the limit of this sequence, $d \in[0,+\infty]$. Assume that $d=+\infty$. Then $\lim _{n \rightarrow \infty} \sqrt[n]{\alpha_{n}}=+\infty$. This contradicts the inequality $\sqrt[n]{\alpha_{n}} \leq \sqrt[n]{C} a$. Therefore, $d \in \mathbb{R} ;$ i. e., the sequence $\left\{\frac{\alpha_{n+1}}{\alpha_{n}}\right\}$ is bounded. The lemma is proved.

Note that the requirement of monotonicity in the second part of the lemma is essential.

Example 1. Suppose that a sequence $\left\{n_{m}\right\} \subset \mathbb{N}$ is such that $n_{m+1}>n_{m}+1,2^{n_{m}}>m$ !, and $\alpha_{n}=(m-1)$ ! for $n_{m-1}<n \leq n_{m}\left(n_{0}=0\right)$ for all $m \in \mathbb{N}$. In this case, the sequence $\left\{\frac{\alpha_{n+1}}{\alpha_{n}}\right\}$ is unbounded, although $\alpha_{n}<2^{n}$. (Indeed, if $n_{m-1}<n \leq n_{m}$, then $\alpha_{n}=(m-1) !<2^{n_{m-1}}<2^{n}$ ).

Assertion 3. Suppose that $k \in \mathbb{N}$ and $\left\{\alpha_{n}\right\}_{n=0}^{\infty} \subset(0,+\infty)$. If the sequence $\left\{\frac{\alpha_{n+k}}{\alpha_{n}}\right\}$ is bounded, then $\alpha_{n} \leq C b^{n}$ for some $b, C \in(0,+\infty)$ and all n. Conversely, if the sequence $\left\{\frac{\alpha_{n+k}}{\alpha_{n}}\right\}$ is monotonic, $C, b \in(0,+\infty)$, and $\alpha_{n} \leq C b^{n}$ for all $n$, then the sequence $\left\{\frac{\alpha_{n+k}}{\alpha_{n}}\right\}$ is bounded.

P r o o f. Suppose that $a \in \mathbb{R}$ is such that $\frac{\alpha_{n+k}}{\alpha_{n}} \leq a$ for all $n$. Let us consider the subsequences $\left\{\beta_{m}^{(r)}\right\}_{m=0}^{\infty}$ of $\left\{\alpha_{n}\right\}$ with $\beta_{m}^{(r)}=\alpha_{m k+r}(r=0,1, \ldots, k-1)$. For all $m$, we have

$$
\frac{\beta_{m+1}^{(r)}}{\beta_{m}^{(r)}}=\frac{\alpha_{(m+1) k+r}}{\alpha_{m k+r}}=\frac{\alpha_{(m k+r)+k}}{\alpha_{m k+r}} \leq a .
$$


Then, according to Lemma 1 , there is a number $C_{r} \in(0,+\infty)$ such that

$$
\beta_{m}^{(r)} \leq C_{r} a^{m}=\frac{C_{r}}{a^{r / k}}(\sqrt[k]{a})^{m k+r}
$$

for all $m$. Setting $C=\max _{0 \leq r \leq k-1} \frac{C_{r}}{a^{r / k}}$ and $b=\sqrt[k]{a}$, we obtain $\alpha_{n} \leq C b^{n}$ for all $n$.

Conversely, suppose that the sequence $\left\{\frac{\alpha_{n+k}}{\alpha_{n}}\right\}$ is monotonic, $C, b \in(0,+\infty)$, and $\alpha_{n} \leq C b^{n}$ for all $n$. If $\lim _{n \rightarrow \infty} \frac{\alpha_{n+k}}{\alpha_{n}}$ is finite, then there is nothing to prove. Assume that $\lim _{n \rightarrow \infty} \frac{\alpha_{n+k}}{\alpha_{n}}=+\infty$. Again, introducing $\beta_{m}^{(r)}=\alpha_{m k+r}(r=0,1, \ldots, k-1)$, we conclude that the sequence $\left\{\beta_{m}^{(r)}\right\}_{m=0}^{\infty}$ is monotonic because

$$
\frac{\beta_{m+1}^{(r)}}{\beta_{m}^{(r)}}=\frac{\alpha_{(m k+r)+k}}{\alpha_{m k+r}}
$$

Moreover,

$$
\beta_{m}^{(r)}=\alpha_{m k+r} \leq C b^{m k+r}=C_{1} b_{1}^{m} \quad\left(C_{1}=C b^{r}, b_{1}=b^{k}\right) .
$$

Hence, according to Lemma 1,

$$
\frac{\alpha_{(m k+r)+k}}{\alpha_{m k+r}}=\frac{\beta_{m+1}^{(r)}}{\beta_{m}^{(r)}} \leq a_{r}
$$

for some $C_{r}, a_{r} \in(0,+\infty)$ and arbitrary $m$. Therefore,

$$
\frac{\alpha_{n+k}}{\alpha_{n}} \leq \max \left\{a_{0}, a_{1}, \ldots, a_{k-1}\right\}=a
$$

for all $n$. The assertion is proved.

Assertion 4. Suppose that operators $B_{1}, \ldots, B_{n}$ act in $X, B_{1}, \ldots, B_{n-1}$ are linear operators with nonempty resolvent sets, $x \in X$, the series

$$
\sum_{m_{1}, \ldots, m_{n}=0}^{\infty} \frac{B_{1}^{m_{1}} \ldots B_{n}^{m_{n}}}{m_{1} ! \ldots m_{n} !} x
$$

converges absolutely, and the following condition holds:

$(v)$ for all $k \in \mathbb{N}$ and a set of natural indices $i_{1}, \ldots, i_{k}$ not exceeding $n$, the expression $B_{i_{1}} \ldots B_{i_{k}} x$ is valid and, if a set $j_{1}, \ldots, j_{k}$ is obtained from the set $i_{1}, \ldots, i_{k}$ by a rearrangement of its elements, then

$$
B_{i_{1}} \ldots B_{i_{k}} x=B_{j_{1}} \ldots B_{j_{k}} x
$$

In this case,

$$
e^{B_{1}} \ldots e^{B_{n}} x=e^{B_{1}+\cdots+B_{n}} x
$$

(both parts of the relation are valid).

P r o o f. Let us first establish the equality

$$
\sum_{m_{1}=0}^{\infty} \frac{B_{1}^{m_{1}}}{m_{1} !} \sum_{m_{2}=0}^{\infty} \frac{B_{2}^{m_{2}}}{m_{2} !} \cdots \sum_{m_{n}=0}^{\infty} \frac{B_{n}^{m_{n}}}{m_{n} !} x=\sum_{m_{1}, m_{2}, \ldots, m_{n}=0}^{\infty} \frac{B_{1}^{m_{1}} B_{2}^{m_{2}} \ldots B_{n}^{m_{n}}}{m_{1} ! m_{2} ! \ldots m_{n} !} x
$$


by induction on $n$. For $n=1$, (6) holds. Assume that, under the conditions of the assertion, equality (6) holds for $n=k-1(k \geq 2)$. Now, let $n=k$. Note that the absolute convergence of series (4) implies the absolute convergence of the series

$$
\sum_{m_{2}, \ldots, m_{n}=0}^{\infty} \frac{B_{2}^{m_{2}} \ldots B_{n}^{m_{n}}}{m_{2} ! \ldots m_{n} !} x .
$$

Taking into account the fact that the operators $B_{1}^{m_{1}}\left(m_{1} \in \mathbb{N}\right)$ are closed, condition $(v)$, and the induction hypothesis, we obtain

$$
\begin{aligned}
& \sum_{m_{1}=0}^{\infty} \frac{B_{1}^{m_{1}}}{m_{1} !} \sum_{m_{2}=0}^{\infty} \frac{B_{2}^{m_{2}}}{m_{2} !} \cdots \sum_{m_{n}=0}^{\infty} \frac{B_{n}^{m_{n}}}{m_{n} !} x=\sum_{m_{1}=0}^{\infty} \frac{B_{1}^{m_{1}}}{m_{1} !} \sum_{m_{2}, \ldots, m_{n}=0}^{\infty} \frac{B_{2}^{m_{2}} \ldots B_{n}^{m_{n}}}{m_{2} ! \ldots m_{n} !} x= \\
& =\sum_{m_{1}=0}^{\infty} \sum_{m_{2}, \ldots, m_{n}=0}^{\infty} \frac{B_{1}^{m_{1}} B_{2}^{m_{2}} \ldots B_{n}^{m_{n}}}{m_{1} ! m_{2} ! \ldots m_{n} !} x=\sum_{m_{1}, m_{2}, \ldots, m_{n}=0}^{\infty} \frac{B_{1}^{m_{1}} B_{2}^{m_{2}} \ldots B_{n}^{m_{n}}}{m_{1} ! m_{2} ! \ldots m_{n} !} x,
\end{aligned}
$$

i. e., equality (6) is proved. Using this equality, we obtain

$$
\begin{gathered}
e^{B_{1}} \ldots e^{B_{n}} x=\sum_{m_{1}, \ldots, m_{n}=0}^{\infty} \frac{B_{1}^{m_{1}} \ldots B_{n}^{m_{n}}}{m_{1} ! \ldots m_{n} !} x=\sum_{s=0}^{\infty} \sum_{m_{1}+\cdots+m_{n}=s} \frac{B_{1}^{m_{1}} \ldots B_{n}^{m_{n}}}{m_{1} ! \ldots m_{n} !} x= \\
=\sum_{s=0}^{\infty} \frac{1}{s !} \sum_{\substack{m_{1}+\ldots+m_{n}=s \\
\left(m_{1}, \ldots, m_{n} \geq 0\right)}} \frac{s !}{m_{1} ! \ldots m_{n} !} B_{1}^{m_{1}} \ldots B_{n}^{m_{n}} x=\sum_{s=0}^{\infty} \frac{\left(B_{1}+\cdots+B_{n}\right)^{s}}{s !} x=e^{B_{1}+\cdots+B_{n}} x .
\end{gathered}
$$

The assertion is proved.

Remark 4. Equality (5) holds if the operators $B_{1}, \ldots, B_{n}$ pairwise commute and the left-hand side of (5) is valid.

Corollary 2. Suppose that $\alpha_{1}, \ldots, \alpha_{n} \in \mathbb{C}, x \in X, A$ is a linear operator acting in $X, \rho(A) \neq \varnothing$, and a series

$$
\sum_{m_{1}, \ldots, m_{n}=0}^{\infty} \frac{\alpha_{1}^{m_{1}} \ldots \alpha_{n}^{m_{n}}}{m_{1} ! \ldots m_{n} !} A^{m_{1}+\cdots+m_{n}} x
$$

converges absolutely. Then,

$$
e^{\alpha_{1} A} \ldots e^{\alpha_{n} A} x=e^{\left(\alpha_{1}+\cdots \alpha_{n}\right) A} x
$$

(both sides of the equality are valid).

To formulate the next assertion, let us introduce some definitions and make a number of assumptions.

Suppose that $\mathscr{L}=\mathscr{L}(p, q)(p>0, q>0)$ is a curve given in the complex plane $(\lambda)$ by the equation

$$
\beta^{2}=2 p \alpha \ln \frac{\alpha}{q} \quad(\alpha=\operatorname{Re} \lambda, \beta=\operatorname{Im} \lambda, \alpha \geq q) ;
$$

Let $G=G(p, q)$ be a domain containing the origin with the boundary $\mathscr{L}$; let the direction of $\mathscr{L}$ be chosen so that the domain $G$ is on the right; and let $A$ be an injective linear operator with domain $D(A)$ dense in $X$ and range $\operatorname{Im}(A) \subset X$. The following estimate for the norm of the resolvent operator $R(\lambda)=R_{A}(\lambda)=(A-\lambda E)^{-1}$ of the operator $A$ in $\bar{G}$ is known:

$$
\|R(\lambda)\| \leq \frac{C_{0}}{(|\lambda|+1)^{\gamma}}
$$


for some $C_{0}>0$ and $\gamma \leq 1$ and all $\lambda \in \bar{G}$.

For $\varphi \in(0, \pi)$, we denote by $\Delta(\varphi)$ the domain in $\mathbb{C}$ that contains the negative real semiaxis and its boundary is $L(\varphi)=L_{1}(\varphi) \cup L_{2}(\varphi)$, where

$$
L_{1}(\varphi)=\left\{\lambda \in \mathbb{C}: \lambda=t e^{i \varphi}, t \geq 0\right\}, \quad L_{2}(\varphi)=\left\{\lambda \in \mathbb{C}: \lambda=t e^{-i \varphi}, t \geq 0\right\} .
$$

Suppose that $\Omega(a, \varphi)=\Delta(\varphi) \cup B(0, a)(a>0, \varphi \in(0, \pi)$, and $B(0, a)$ is the open disk of radius $a$ centered at the origin), and the direction of $\Gamma(a, \varphi)=\partial \Omega(a, \varphi)$ is chosen so that $\Omega(a, \varphi)$ is on the right. Given $p$ and $q$, we chose $a$ and $\varphi$ so that $\overline{\Omega(a, \varphi)} \subset G(p, q)$.

Under these assumptions, the authors studied [5] the operator functions

$$
\begin{aligned}
& f(A)=-\frac{1}{2 \pi i} A^{n} \int_{\Gamma(a, \varphi)} \frac{f(\lambda)}{\lambda^{n}} R(\lambda) d \lambda, \\
& \widetilde{f}(A)=-\frac{1}{2 \pi i} \overline{\int_{\Gamma(a, \varphi)} \frac{f(\lambda)}{\lambda^{n}} R(\lambda) d \lambda A^{n}}
\end{aligned}
$$

constructed on the basis of corresponding scalar functions $f(\lambda)$ continuous in $\mathbb{C} \backslash \Omega(a, \varphi)$ and analytic in $\mathbb{C} \backslash \overline{\Omega(a, \varphi)}$; in addition, for every such function $f$ there exist $C \in(0,+\infty)$ and $\sigma \in \mathbb{R}$ such that

$$
|f(\lambda)| \leq C|\lambda|^{\sigma}
$$

for all $\lambda \in \mathbb{C} \backslash \Omega(a, \varphi)$. The number $n \in \mathbb{N} \cup\{0\}$ in (10) and (11) is chosen so that $\sigma-n-\gamma<-1$.

It was proved that the right-hand sides of these representations are independent of such $n$, the operator functions $f(A)$ and $\widetilde{f}(A)$ are densely defined, $\widetilde{f}(A) \subset f(A)$, and the functions coincide if one of them is continuous.

We can take the function $e^{-t \lambda}(t>0)$ as the function $f$ and consider two operator functions, one of which is given by series according to formula (1) and the other is given by relations (10) and (11) for $n=0$ (these relations yield the same result because their right-hand sides are continuous). Denoted by $\left(e^{-t A}\right)_{I}$ the function given by formulas (10) and (11).

Lemma 2. Let $\sigma \in \mathbb{R}, \sigma-\gamma<-1$, and let a function $f$ be continuous in $\mathbb{C} \backslash \Omega(a, \varphi)$, analytic in $\mathbb{C} \backslash \overline{\Omega(a, \varphi)}$ and such that (12) holds for some $C \in(0,+\infty)$ and all $\lambda \in \mathbb{C} \backslash \Omega(a, \varphi)$.

Then

$$
\int_{\Gamma(a, \varphi)} f(\lambda) R(\lambda) d \lambda=\int_{\mathscr{L}(p, q)} f(\lambda) R(\lambda) d \lambda
$$

The proof of this lemma is similar to the proof of [3, Lemma 1].

Assertion 5. Let a curve $\mathscr{L}_{t}(t>0)$ be given by the equation $\beta^{2}=2 t p \alpha \ln \frac{\alpha}{q t}(\alpha=\operatorname{Re} \lambda$, $\beta=\operatorname{Im} \lambda$, and $\alpha \geq q t)$, and let $n(t) \in \mathbb{N}$ satisfy the inequality

$$
t p-n(t)-\gamma<-1
$$

Then

$$
\begin{gathered}
\left.e^{t A}\left(e^{-t A}\right)_{I}\right|_{D\left(A^{n(t)}\right)}=\left.E\right|_{D\left(A^{n(t)}\right)}, \\
\left(e^{-t A}\right)_{I} e^{t A}=\left.E\right|_{D\left(e^{t A}\right)} .
\end{gathered}
$$


P r o o f. Let us first consider the case $t=1$. Note that $\mathscr{L}_{1}=\mathscr{L}$. Denote by $n_{1}^{0}$ the value $n(1)$. The following equalities hold for $x \in D\left(e^{A}\left(e^{-A}\right)_{I}\right)$ :

$$
e^{A}\left(e^{-A}\right)_{I} x=-\frac{1}{2 \pi i} \sum_{n=0}^{\infty} \frac{A^{n}}{n !} \int_{\mathscr{L}} e^{-\lambda} R(\lambda) d \lambda x=-\frac{1}{2 \pi i} \lim _{n \rightarrow \infty} \sum_{k=0}^{n} \frac{A^{k}}{k !} \int_{\mathscr{L}} e^{-\lambda} R(\lambda) d \lambda x .
$$

For every $n \in \mathbb{N}$, consider $n_{1} \in \mathbb{N}$ satisfying the inequality $n-n_{1}-\gamma<-1$. Then, according to $[4$, Theorem 9],

$$
\sum_{k=0}^{n} \frac{A^{k}}{k !}=-\frac{A^{n_{1}}}{2 \pi i} \int_{\mathscr{L}} \sum_{k=0}^{n} \frac{\lambda^{k-n_{1}}}{k !} R(\lambda) d \lambda
$$

i. e., according to [5, Theorem 3],

$$
\sum_{k=0}^{n} \frac{A^{k}}{k !} \int_{\mathscr{L}} e^{-\lambda} R(\lambda) d \lambda=A^{n_{1}} \int_{\mathscr{L}} \sum_{k=0}^{n} \frac{\lambda^{k-n_{1}}}{k !} e^{-\lambda} R(\lambda) d \lambda .
$$

For the functions $f_{n}(\lambda)=\sum_{k=0}^{n} \frac{\lambda^{k}}{k !} e^{-\lambda}$ in (10) and (11), we can take an arbitrary $\sigma$ from (12). Thus, the right-hand side of (16) is independent of $n_{1} \in \mathbb{N} \cup\{0\}$. Therefore, for $x \in D\left(A^{n_{1}^{0}}\right)$, we have

$$
A^{n_{1}} \int_{\mathscr{L}} \sum_{k=0}^{n} \frac{\lambda^{k-n_{1}}}{k !} e^{-\lambda} R(\lambda) d \lambda x=A^{n_{1}^{0}} \int_{\mathscr{L}} \sum_{k=0}^{n} \frac{\lambda^{k-n_{1}^{0}}}{k !} e^{-\lambda} R(\lambda) d \lambda x=\int_{\mathscr{L}} \frac{\lambda^{k-n_{1}^{0}}}{k !} e^{-\lambda} R(\lambda) d \lambda A^{n_{1}^{0}} x,
$$

i.e.,

$$
e^{A}\left(e^{-A}\right)_{I} x=-\frac{1}{2 \pi i} \lim _{n \rightarrow \infty} \int_{\mathscr{L}} \sum_{k=0}^{n} \frac{\lambda^{k-n_{1}^{0}}}{k !} e^{-\lambda} R(\lambda) d \lambda A^{n_{1}^{0}} x
$$

whenever this limit exists. Let us establish the existence of this limit and find its value using the Lebesgue (dominated convergence) theorem on passing to the limit under the integral sign. Let us check the conditions of this theorem.

Let

$$
H_{n}(\lambda)=\sum_{k=0}^{n} \frac{\lambda^{k-n_{1}^{0}}}{k !} e^{-\lambda} R(\lambda)
$$

and let $\lambda=\alpha+i \beta \in \mathscr{L}$ be arbitrary. Then

$$
\lim _{n \rightarrow \infty} H_{n}(\lambda)=\sum_{n=0}^{\infty} \frac{\lambda^{n-n_{1}^{0}}}{n !} e^{-\lambda} R(\lambda)=\lambda^{-n_{1}^{0}} R(\lambda)
$$

(the limit and the convergence of the series are considered with respect to the operator norm). Let us show that the sequence $\left\{\left\|H_{n}(\lambda)\right\|\right\}$ is dominated by a Lebesgue integrable function in $\mathscr{L}$ :

$$
\left\|H_{n}(\lambda)\right\| \leq \sum_{k=0}^{n} \frac{|\lambda|^{k-n_{1}^{0}}}{k !} e^{-\alpha}\|R(\lambda)\| \leq \frac{C_{0}|\lambda|^{-n_{1}^{0}} e^{|\lambda|-\alpha}}{(|\lambda|+1)^{\gamma}} \leq C_{0} C_{1}|\lambda|^{-n_{1}^{0}-\gamma} e^{|\lambda|-\alpha},
$$

where $C_{1}=\sup _{\mu \in \mathscr{L}}\left(\frac{|\mu|}{|\mu|+1}\right)^{\gamma}$ (the function $\left(\frac{|\mu|}{|\mu|+1}\right)^{\gamma}$ is continuous in $\mathscr{L}$, has a finite limit at infinity and, therefore, is bounded in $\mathscr{L}$ ). Using (8), we obtain that

$$
|\lambda|-\alpha=\sqrt{\alpha^{2}+\beta^{2}}-\alpha=\frac{\beta^{2}}{\sqrt{\alpha^{2}+\beta^{2}}+\alpha} \leq \frac{2 p \alpha \ln \frac{\alpha}{q}}{2 \alpha}=p \ln \frac{\alpha}{q}
$$


i. e.,

Hence,

$$
e^{|\lambda|-\alpha} \leq\left(\frac{\alpha}{q}\right)^{p} \leq\left(\frac{|\lambda|}{q}\right)^{p}
$$

where $\bar{C}=\frac{C_{0} C_{1}}{q^{p}}$. By (13) for $t=1$, the integral $\int_{\mathscr{L}}|\lambda|^{p-n_{1}^{0}-\gamma}|d \lambda|$ converges. By the Lebesgue theorem,

$$
-\frac{1}{2 \pi i} \lim _{n \rightarrow \infty} \int_{\mathscr{L}} H_{n}(\lambda) d \lambda=-\frac{1}{2 \pi i} \int_{\mathscr{L}} \lambda^{-n_{1}^{0}} R(\lambda) d \lambda=A^{-n_{1}^{0}}
$$

(the limit is considered with respect to the operator norm). Therefore,

$$
e^{A}\left(e^{-A}\right)_{I} x=A^{-n_{1}^{0}} A^{n_{1}^{0}} x=x,
$$

and (14) is proved.

Let us show that (15) holds. For $x \in D\left(e^{A}\right) \subset \stackrel{n}{n=0}_{\infty}^{\infty} D\left(A^{n}\right)$, in view of continuity of the operator $\left(e^{-A}\right)_{I}$, we have

$$
\left(e^{-A}\right)_{I} e^{A} x=\left(e^{-A}\right)_{I} \lim _{n \rightarrow \infty} \sum_{k=0}^{n} \frac{A^{k} x}{k !}=-\frac{1}{2 \pi i}\left(e^{-A}\right)_{I} \int_{\mathscr{L}} \sum_{k=0}^{n} \frac{\lambda^{k-n_{1}}}{k !} R(\lambda) d \lambda A^{n_{1}} x
$$

where $n_{1}$, as before, satisfies inequality (13) for $t=1$. Arguing similarly to the proof of formula (14), we obtain (15). Thus, the assertion holds for $t=1$.

Let us now consider an arbitrary $t>0$. The mapping $\mu=t \lambda$ takes the curve $\mathscr{L}$ to the curve $\mathscr{L}_{t}$ and the domain $G=G_{1}$ to the domain $G_{t} \ni 0$ such that $\partial G_{t}=\mathscr{L}_{t}$. In addition, $\rho(t A)=t \rho(A)$ $(\rho(A)$ and $\rho(t A)$ are the regular sets of the operators $A$ and $t A$, respectively) and the estimate for $\left\|R_{t A}(\lambda)\right\|$ in $G_{t}$ coincides with the estimate $(9)$ for $\left\|R_{A}(\lambda)\right\|$ in $G$ with certain constant $C_{t}$ instead of $C_{0}$. The analysis of the proof for $t=1$ shows that formulas (14) and (15) remain valid for $t>0$ under condition (13). The assertion is proved.

Corollary 3. If $t>0$ and the operator $e^{t A}$ is closed, then it is invertible and $\left(e^{t A}\right)^{-1}=\left(e^{-t A}\right)_{I}$.

The corollary follows from (14), (15), and the fact that if a closed operator coincides with a continuous operator on a dense set, then they coincide in the entire space.

Example 2. Let $X=L_{p}[1,+\infty)$ and $A x(t)=t x(t)(x \in X)$. Let us show that $\mathcal{D}\left(e^{A}\right)=\{x \in$ $\left.X: e^{t} x \in X\right\}$ and the equality $e^{A} x=e^{t} x$ holds for $x \in \mathcal{D}\left(e^{A}\right)$.

Let $x \in X$ and $e^{t} x \in X$. Let us establish that $x \in \mathcal{D}\left(e^{A}\right)$ and $e^{A} x=e^{t} x$. To this end, we have to prove that the series $\sum_{n=0}^{\infty} \frac{t^{n}}{n !} x$ converges in $X$ to $e^{t} x$, i. e., that

$$
\left\|e^{t} x-\sum_{k=0}^{n} \frac{A^{k} x}{k !}\right\|=\left\|e^{t} x-\sum_{k=0}^{n} \frac{t^{k} x}{k !}\right\| \underset{k \rightarrow \infty}{\longrightarrow} 0 .
$$

Since $\int_{1}^{+\infty} e^{p t}|x(t)|^{p} d t<+\infty$, there exists a function $\alpha(t)$ defined on $[1,+\infty)$ such that $\alpha(t) \geq \alpha_{0}$ for some $\alpha_{0}>0, \alpha(t) \underset{t \rightarrow \infty}{\longrightarrow}+\infty$ (in particular, we can take a continuous positive function $\alpha$ with infinite limit at $+\infty)$, and $\int_{1}^{+\infty}\left|\alpha(t) e^{t} x(t)\right|^{p} d t<+\infty$. Then

$$
\left\|e^{t} x-\sum_{k=0}^{n} \frac{t^{k} x}{k !}\right\|=\left\|\frac{1-e^{-t} \sum_{k=0}^{n} t^{k} / k !}{\alpha} \alpha e^{t} x\right\| \leq \sup _{t \geq 1} \frac{1-e^{-t} \sum_{k=0}^{n} t^{k} / k !}{\alpha(t)}\left\|\alpha e^{t} x\right\|=\gamma_{n}\left\|\alpha e^{t} x\right\| .
$$


Let us show that

$$
\gamma_{n}=\sup _{t \geq 1} \frac{1-e^{-t} \sum_{k=0}^{n} t^{k} / k !}{\alpha(t)} \underset{n \rightarrow \infty}{\longrightarrow} 0 .
$$

Take an arbitrary $\varepsilon>0$. Since $\alpha(t) \underset{t \rightarrow \infty}{\longrightarrow}+\infty$, there is a number $\Delta>1$ such that $1 / \alpha(t)<\varepsilon$ for all $t \geq \Delta$; i.e.,

$$
\frac{1-e^{-t} \sum_{k=0}^{n} t^{k} / k !}{\alpha(t)} \in[0, \varepsilon)
$$

for all $t \geq \Delta$ and $n \in \mathbb{N}$. Since the power series $\sum_{n=0}^{\infty} t^{n} / n$ ! uniformly converges to $e^{t}$ on $[1, \Delta]$, the sequence of functions

$$
\left\{\frac{1-e^{-t} \sum_{k=0}^{n} t^{k} / k !}{\alpha(t)}\right\}
$$

uniformly tends to zero on $[1, \Delta]$. Hence, there exists a number $N$ such that

$$
\frac{1-e^{-t} \sum_{k=0}^{n} t^{k} / k !}{\alpha(t)}<\varepsilon
$$

for all $t \in[1, \Delta]$ and $n>N$. Thus, $\gamma_{n} \leq \varepsilon$ for all $n>N$, i. e., $\gamma_{n} \underset{n \rightarrow \infty}{\longrightarrow} 0$ and, consequently,

$$
\sum_{n=0}^{\infty} \frac{t^{n}}{n !} x=e^{t} x \quad \text { in } \quad X
$$

Conversely, suppose that $x \in \mathcal{D}\left(e^{A}\right)$ and $e^{A} x=y \in X$, i. e.,

$$
S_{n}=\sum_{k=0}^{n} \frac{t^{k}}{k !} x \underset{n \rightarrow \infty}{\longrightarrow} y \quad \text { in } \quad X .
$$

Then, there exists a subsequence $\left\{S_{n_{k}}\right\}$ of $\left\{S_{n}\right\}$ such that

$$
S_{n_{k}}(t) \underset{k \rightarrow \infty}{\stackrel{\text { a. e. }}{\longrightarrow}} y(t) .
$$

But

$$
S_{n}(t) x(t) \underset{n \rightarrow \infty}{\longrightarrow} e^{t} x(t)
$$

at every point $t \geq 1$; i. e., $y=e^{t} x$ in $X$. Since $e^{t} x \in X$, we have $e^{A} x=e^{t} x$.

Note that equality (7) holds for the operator $A$ if

$$
x, e^{\alpha_{n} t} x, e^{\left(\alpha_{n-1}+\alpha_{n}\right) t} x, \ldots, e^{\left(\alpha_{1}+\cdots+\alpha_{n}\right) t} x \in X .
$$

\section{Conclusion}

We have considered some natural properties of exponential operator defined by power series (Corollary 1 and Assertion 4). The main result of the paper is the connection (under certain conditions) of the exponential operator $e^{A}$ in the form of power series with the exponential function $e^{-A}$ defined on the basis of the Cauchy integral formula (Assertion 5). These facts may give an impulse to obtaining further results on functional calculus of operators. 


\section{REFERENCES}

1. Balakrishnan A. V. Fractional powers of closed operators and the semigroups generated by them. Pacific J. Math., 1960. Vol. 10, No. 2. P. 419-437. URL: https://projecteuclid.org/euclid.pjm/1103038401

2. Dunford N., Schwartz J. T. Linear Operators Part I: General Theory. New York: Interscience Publishers, 1958. $858 \mathrm{p}$.

3. Korkina L.F., Rekant M.A. An extension of the class of power operator functions. Izvestiya Uralskogo gosudarstvennogo universiteta (Matematika $i$ mekhanika) [Bulletin of the Ural State University (Mathematics and Mechanics)], 2005. No. 38. P. 80-90. (in Russian) URL: http://hdl.handle.net/10995/24591

4. Korkina L.F., Rekant M. A. Some classes of functions of a linear closed operator. Proc. Steklov Inst. Math., 2012. Vol. 277, Suppl. 1. P. 121-135. DOI: 10.1134/S0081543812050124.

5. Korkina L.F., Rekant M. A. Properties of mappings of scalar functions to operator functions of a linear closed operator. Trudy Inst. Mat. i Mekh. UrO RAN [Proc. of Krasovskii Institute of Mathematics and Mechanics of the UB RAS], 2015. Vol. 21, No. 1. P. 153-165. (in Russian) URL: http://mi.mathnet.ru/eng/timm/v21/i1/p153

6. Krein S.G. Lineinye differentsial'nye uravneniya $v$ banakhovom prostranstve [Linear Differential Equations in Banach Space]. Moscow: Nauka, 1967. 464 p. (in Russian)

7. Lusternik L. A., Sobolev V.J. Elements of functional analysis. Delhi: Hindustan Publishing Corpn., 1974. 376 p.

8. Rudin W. Functional Analysis. New York: McGraw-Hill, 1973. 397 p. 